this I reasoned that Mr. R—'s failure to entirely empty the bowel was due to the want of solidity of the motion not giving the bowel anything to contract upon. I therefore ordered him occasional doses of lime water, to be taken sufficiently often to cause a formed motion. The result was very satisfactory. The bowel contracted on and expelled the solid motion completely. I think, in our desire to keep the bowels regular, we overlook the disadvantages of fluid instead of a solid motion."

Before concluding this lecture, I will mention a plan which I have found of very great service either in cases of cardiac disease, in fevers, or in conditions of extreme weakness where the feebleness of the heart made it very advisable that the patient should use as little exertion as possible. It frequently happens that patients strongly object to use a bed-pan, and assert, sometimes with a considerable amount of truth, that they are unable to empty the bowels satisfactorily in the recumbent posture. A great deal of the risk connected with the use of a commode arises from the fact that when they use it they generally have to get out of bed and into it again. In these cases I adopt a plan, which I suppose many others have used also, but which I have never seen described. It is to have a strong platform made of rough wood, of such a height that the commode, when placed upon it, is level with the bed. A large, strong packing.box, sawn to the proper height, may answer, or, if this is not at hand, any carpenter can put together a rough but strong construction of the kind needed. If nothing else is at hand, the drawer of a ward. robe turned upside down will do, but it must be supported from below by books or pillows piled up within it, so as to prevent the bottom of it from breaking through, as usually it is not strong enough to support the weight. The commode being pushed close to the bed. side, the patient has simply to slew himself round upon it. The body is raised, and the legs are allowed to hang down. After the bowels have been evacuated, he is rolled round on his side into bed. During the whole process no change is ever made in the level of the patient, the only alteration being in the relative position of the trunk and legs with reference to his pelvis. Perhaps some of the new devices for raising the patient bodily from the bed might be better than this, but generally they are not at hand, and the plan which I have mentioned can always be followed, as some kind of platform sufficiently strong to serve the purpose can be made with boards, boxes, or footstools in every house.

In this lecture I have not attempted to go over anything like the whole field which its title would cover, because I found this to be impossible in the course of the time at my disposal. I have therefore attempted to deal only with such points as have more of a practical than a theoretical interest. In concluding it, I thank you most sincerely, $\mathrm{Mr}$. President and gentlemen, for the great honour you have done me in asking me to deliver this lecture, and to beg you to forgive the deficiencies in it, of which no one is more conscious than myself.

\section{SOME CASES OF BONE ABSCESS.}

BY WILLIAM HENRY BATTLE, F.R.C.S.,

SENIOR ASSISTANT SURGEON TO THE ROYAL FREE HOSPITAL AND TO THE EAST LONDON HOSPITAL FOR CMIMDREN.

THE fire cases which follow are examples of suppuration commencing in the interior of bones. In two it was necessary to let out the pus from the interior of the bone by an operation, and in three the pus had already partly escaped from the bone under or beyond the periosteum before operation was performed. They are interesting from the contrast which they present, one to the other, in their symptoms, from the difficulty in diagnosis and treatment, and because it is not of ten that we are able to compare a similarseries. The amount of pain to which the presence of the pus gave rise varied in the individual cases; it was not dependent upon the size of the abscess, but upon the structure of the bone in which it commenced, and the ease or otherwise with which it was able to escape from the bone-in other words, on "the tension." For permission to treat and publish the notes of the cases which were in St. Thomas's Hospital I am indebted to Mr. Sydney Jones. I desire also to thank Mr. Barrow for per- mission to use the notes of the case which was admittea under his care at the Royal Free Hospital.

CASE 1. Abscess of upper end of the tibia; operation; recovery. - H. M — aged fourteen, at school, was admitted into St. Thomas's Hospital June 6th, under the care of Mr. Sydney Jones, and discharged Aug. 1st, 1888. Three weeks before the patient got wet through. The following day the inner side of the right knee became painful. He went about for a week, but a fortnight before admission he was in bed suffering from severe pain in the leg, which prevented sleep. Between five and six years ago he says that he had an abscess on the bone in his right leg, which was operated on, some dead bone being removed. About two years afterwards an abscess recurred in the same situation, which was again operated on in the Shadwell Children's Hospital, some more dead bone being removed. The patient, a thin delicate-looking boy, complained of pain in his leg. There is a white scar six inches long over the upper half of the outer side of the right tibia, resulting from previous operation. Over the inner head of the same bone there is a hard, tender swelling measuring from three to four inches. Two inches below the patella on the right side the circumference is about two inches greater than at a corresponding. point in the opposite limb. There are increased heat, great tenderness, and pain much worse at night. A Macintyre splint was applied and an ice-bag over the knee.-June 14 th : The affected limb gives much pain at times, chiefly at night, the patient being very restless with it. Lead lotion was applied. Last night a morphia injection was given, but he slept only half an hour. He is frequently found to be crying with the pain.-Operation: On June 20 th an incision about three inches long was made in the upper part of the leg on the inner side, another across the upper part of that, and the periosteum, which was much thickened, incised. On dividing this towards the inner side pus sprang into the wound, showing the excessive tension. at which it had been kept. There was a large cavity which occupied most of the head of the tibia and was bounded with hard bone, the inner surface being filled in with dense fibrous tissue. To expose the cavity fully it was necessary to chisel away some bone. There was no granulation tissue in the cavity, which was washed out with chloride of zinc ( $40 \mathrm{gr}$. to $1 \mathrm{oz}$.) carbolic solution ( 1 in 40), and plugged with strips of lint soaked in carbolic oil. There was a good deal of pain at the earlier dressings, but on July 2 nd the wound looked better and a fair amount of pus came away; the quantity was slight on the $11 \mathrm{th}$, and the wound gradually closed, but on the 25 th was reopened to some extent. Temperature on the 24 th $996^{\circ}$. On the 26 th only slight discharge was coming away and the wound was closed firmly by Aug. 1st.

The history of this patient is one not infrequent in bone abscess, there having been previous attacks of ostitis followed by necrosis. The suppuration was more acute than is usual in central abscess of bone, and the pain extremely severe, so that from want of sleep and inability to take food the boy was becoming pale, worn, and thin. It illustrates the effect of the tension of fluid confined in dense resisting structures in the production of pain, for although the wall of the abscess was not completely formed of bone, the fibrous tissue which completed the cavity was of such extreme thickness and density that, although it probably permitted of the somewhat unusual size of the abscess, it gave so slowly that the tension was very high. Of course in saying that the abscess was of somewhat unusual size for one which had not nearly reached the periosteum of the bone in which it began, $I$ do not mean that it enters into the same category as the large expanded cavity mentioned by Stanley, ${ }^{1}$ which contained over sixteen ounces of fluid, and which the patient emptied at regular times of the day by the removal of a plug of wood and tow, which blocked an opening into it.

CASE 2. Circumseribed abscess of lower end of tibia; trephining; primary union; recovery.-J. $\mathbf{M}-$, aged sixteen, a tailor's apprentice, sent to me from Bedford, was admitted to the Royal Free Hospital on May 11th, 1889. His mother states that he had suffered from pain in the leg for from five to six years, and had hurt it three times : once a man trod on it, once it was struck by a hockey stick, and once an iron spike ran into it. He himself said that it had been worse since he knocked it against a tree about four years ago, two or three days after which it became very 
painful, but there was no swelling of the limb or redness. During this time he has had attacks of pain at intervals of two or three weeks of a severe character, so that he has been unable to eat or sleep, but has stayed, crying, in bed, anable to bear the bedclothes to touch it. There has occasionally been pain between these attacks, worse in damp weather, but as a rule he is able to walk about without any inconvenience. About twelve months ago he first noticed the left ankle was larger than the right, and this swelling has increased slightly. The lower end of the left tibia was found enlarged from the tip of the malleolus upwards for a distance of one inch and a half to two inches. The surface is smooth, the skin over it normal in appearance, but hotter than elsewhere. Tenderness is complained of on deep firm pressure, especially on the anterior aspect. The circumference of the limb at the most prominent part of the internal malleolus is about three-eighths of an inch greater than on the right side. The tibire are equal in length and the movements of the ankle-joints equally free and painless.

As the symptoms were so very slight on admission it was decided to keep him quiet in bed and administer iodide of potassium and syrup of the iodide of iron, and apply tincture of iodine locally. The general temperature was normal, excepting during a short attack of tonsillitis, but the surface temperature over the swelling above the left ankle was found to be two degrees higher than over a corresponding point on the opposite limb. Sometimes this difference was more marked. May 31st: Right, $81^{\circ}$; left, $885^{\circ}$.-June 6 th : Right, $84^{\circ}$; left, $89 \cdot 5^{\circ}$.-7th : Right, $91^{\circ}$; left, $93 \cdot 5^{\circ}$. -8 th : Right, $82.5^{\circ}$; left, $88^{\circ}$. On the morning of June 3rd he awoke complaining of a jumping pain in the left ankle, shooting up the leg; it kept him awake for an hour. The ankle, increased about a quarter of an inch in circumference, became hot and tender, but there was no rise in the general temperature. On June 8th the limb was rendered bloodless in the usual way, and the area of operation again carefully washed with antiseptics. The position of the supposed abscess was marked on the skin. A longitudinal incision was then made behind the more prominent part of the swelling on the inner surface of the tibia, and another from the centre of this forwards. Flaps were raised, including the greatly thickened periosteum, the bone exposed, and the trephine applied. After sawing for a depth of nearly half an inch the removal of the circle of bone opened an abscess cavity, in which lay a quantity of pus surrounded with sof $t$ granulation tissue. The lower boundary of this extended to the epiphysial line; upwards a probe passed for some distance. The incision was prolonged upwards, more bone removed with a chisel, and the whole length of the cavity exposed fully. It was about two inches in length, was narrow, and bounded by hard, dense bone. It was scraped out with the sharp spoon, mopped out with chloride of zinc forty grains to the ounce, and perchloride of mercury solution. The edges of the periosteum and skin were respectively brought together with catgut sutures. One small bloodvessel required twisting on removal of the Esmarch the cavity was allowed to fill with blood-clob, perchloride dressings were applied, and the leg and ankle fixed with an anterior plaster-of-Paris splint. The temperature rose to $100.4^{\circ}$ on the evening of the 9 th, and there was some pain on the evenings of the 8 th and $9 \mathrm{ch}$, but this did not recur, and the temperature was afterwards always normal. The first dressing was changed a fortnight after the operation, when the wound had closed excepting for a small granulating surface in the upper part about half an inch long. This soon healed, and he left the hospital on July 9th quite well. The surface temperature was taken before he went, and was a degree higher over the scar than on the other side. Microscopical examination of the granulation tissue removed from the cavity showed the ordinary structure of granulation tissue with some scattered nodules of closely packed cells surrounding a giant cell. No tubercle bacilli could be found.

"This boy has not had any attack of pain since he left the hospital, and is in good health.

This abscess presented the signs which we are accustomed to look for in chronic circumscribed abscess of bone, localised paroxysmal pain of long duration, marked tenderness and some enlargement of the area affected. Further, there was the increased local heat, as proved by the surface thermometer, quite independently of any general rise of temperature. So far as I have been able to discover, we have few, if any, accurate records of the actual differences of temperature which may be met with in chronic abscess of bone, and it is posible that in this there is means of rendering a more accurate diagnosis possible between the various localised bone inflammations and circumscribed abscess. The mode of treatment of the abscess cavity after evacuation of its contents is an advance on that in which the drainage-tube is used. After the wound is closed, healing commences at once between the opposed soft structures, and the aseptic clot which fills the cavity commences to organise. Time is saved, for the tube may cause suppuration. ${ }^{2}$ It would be out of place to discuss here the question whether Bloonifield or Brodie is entitled to credit for our instruction in the diagnosis and treatment of chronic abscess of bone, I would refer those interested in the question to Mr. Bryant's lectures on "Tension."

CASE 3. Small abscess in shaft of tibia.-J. B-, aged twenty-two, a brushmaker, living at Chesham, was admitted to St. Thomas's Hespital May 17th, and discharged July24th, 1886. His mother died from phthisis; six brothers and sisters died as infants. He had always been strong and healthy. Six years ago the man noticed a slight thickening over the upper part of the right tibia. This remained stationary, causing some pain at intervals, preventing work at times, until five months ago, when it began to increase in size and canse more pain, so that he was unable to work at all. Three months ago he noticed that the bone over the centre of the swelling was quite s oft. There was no breaking of the skin. He had been in a country hospital, but nothing had been done. From there he was sent to a convalescent home. At the convalescent home he was unable to get about like other patients, but used to lie on the sofa all day, on account of the pain in his leg. This pain, of an aching character, had been worse at night time. On admission, he complained of a swelling, over the upper third of the righo tibia. At the centre of the swelling there was a circular depression to be felt in the tibia as large as half-a-crown, bounded by a sharp edge of bone. There was not much tenderness round this aperture. Limited fluctuation could be felt; skin somewhat discoloured over this. General health good. Evening temperature $99 \cdot 6^{\circ}$. May 20th: Under ether an incision was made into this swelling. A cavity in the bone was scraped out with a sharp spoon, the use of $a$ chisel being required to open it up. The sinus ran into the medulla and terminated in a small deposit of granulation tissue surrounded by hard bone. Pus of a caseous character was found on incising the skin. The cavity gradually closed by granulation, and when the patient was heard of some months later he was in good health and able to work, having lost his pain.

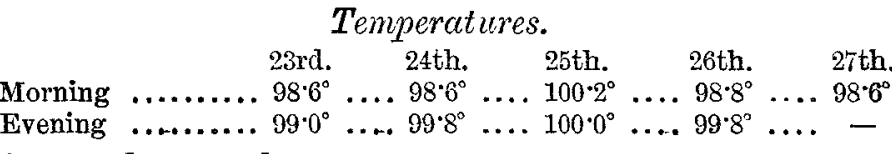

Afterwards normal.

Circumscribed abseess of the shaft of the tibia is a much less frequent disease than abscess of the extremities of the bone. The mischief in this case was so limited that it was a subject of remark at the time of operation. For years the man had been unable to do his work on accoun of the attacks of pain in the bone, which compelled him to rest. There is a great resemblance between this case and one described by Hey of Leeds. ${ }^{4}$ A young lady had a feverish attack, followed by severe pain in the shaft of the tibia. She came under his care six weeks later, and at the operation pus was found under the periosteum, bone was removed, and the cavity afterwards cleared out with a strong knife.

CASE 4. Abscess in the lower end of the femur, producing effusion in the linee joint; operation; recovery. - A boy, aged eleven, was admitted to Marsden ward of the Royal Free Hospital, under the care of Mr. Barrow, on July 3lst, 1889 , suffering from caries of the right os calcis of nine months' duration. He had al ways been a delicate child, and there was a strong history of phthisis on the father's side. Mr. Barrow operated for the disease of the os calcis on Aug. 9th, and the cavity was afterwards dressed so as to heal from the bottom. On the 30 th the patient complained of pain in the left knee, and when closely questioned said that he had pain in it for the first time in June, and had had it on two or three occasions since; once it was so bad he could hardly walk. The pain of which he complained was dull and aching, but not severe. I found the knee enlarged, and

2 J. Lloyd, Provincial Medical Journal, 1890, p. 198.

* Practical Observations in Surgery, third edition, London, 1814. 
this enlargement was due to effusion in the joint, the measurement being half an inch more than that of the oppo. site side. There was tenderness on pressure just above the inner condyle, but this was not very marked. He was perspiring freely at night time, but was otherwise doing well. A back splint was applied to the limb and tincture of iodine to the joint The temperature was rather higher at night about this time, and rose to $102.2^{\circ}$ on the 10th of September. The effiasion in the joint was now almost gone, but he complained of paia behind the knee when an attempt was made to fully extend the limb. Un the 11 th it was possible to feel thickening over both condyles, especially behind, with fulness in the popliteal space. These parts were also tender. A Macinty re splint with evaporating lotion was applied. Being in charge of the case at this time, it was my opinion that the buy had a tubercular centre in the popliteal space ab ut the epiphysial line, and that the effusion in the knee joint was due to the spread of inflammation from that. It was decided to $\epsilon \mathrm{x}$ plore the popliteal space; for, although there wa; no flactuation in the thickening to be felt there, the rise in temperature occurring at the time of the effusion in the knee, taken together with the increasing evidence of disease in the popliteal space, made it appear desirable to provide a way of escape for inflammatory products other than towards the knee-joint. The effusion had subsided, but measurement showed an inch larger on the left side on Sept. 18th, when an incision was made into the popliteal space from the inner side of the thigh just above the internal condyle. The incision was carried down to the hone, and a director pushed under the periosteum to the popliteal surface of the femnr, when pus welled up by the side of it The thickened peiosteam was raised with the elevator from the popliteal surface, and the pus was found to be escaping from an opening at the line of the epiphysis about the centre of the space. A probe passed through whis went into the condyles. The opening was enlarged, and access more readily obtained for the finger, by chiselling a groove in the lower end of the diaphysis of the femur. The finger was then passed into a large cavity in the condyles, which contained some pus and granulation tissue, which was scraped or washed away This cavi:y was of very large siz, appearing to be bounded only by the more compact tissue of the bone, and the forefinger in the condyles seemed to be separated from the thumb over the joint only by a thickness of tissues of about a quarter of un inch; a mere shell of bone appeared to remain. The whole was washed out with chloride of zinc (forty grains to the ounce), a drainage-tube inserted, the wound partly closed with sutures, and an anterior plaster splint applied to the front of the flexed limb over perchloride dressings. In the evening he complained of severe pain, and tincture of opium was given. On Oet. 30:h Mr. Barrow scraped out the sinus in the os calcis again, and removed more tubercular material. On Nov. 8th the sinus above the left knee remaining from the drainage-tube had healed, and there had $b$ sen no further effusion in the knee-joint. In consequence of the granulating cavity in the os calcis taking a long time to close he did not leave until Jan. $27 \mathrm{ch}, 1890$. The sinus above the knee remained closed, but during the year after he left reopened for a time. There has been no further trouble with the knee, and he is able to run about

It is probable that the operation for this patient saved the limb, for had the contents of the abscess found their way in to the knee-joint, nothing short of amputation would have been possible, the almost complete destruction of the incerior of the articular end of the femur would have prevented the trial of conservative treatment. Abscess in the lower end of the femur is rare, but is sometimes met with when operation is performed for disease of the joint, and primary disease of the bones entering into this articalation is not uncommon. We can, however, rarely meet with the case at a stage sufficiently early to make operation on the bone likely to be of much use in arresting the disease of the joint. There has been considerable advance made during the last few years in the treatment of diseases of the articular ends of the long bones. It is recognised that occasionally better results are obtained by the performance of an operation which appears adequate to the removal of the disease, than in the completion of one of the set operations of the text-books. In no part of the body is this better exemplied than in the opper end of the femur. In the discussion at the Clinical Society on some cases brought forward by Mr. Symonds ${ }^{3}$ this was fully exemplified. In a girl with suppuration in the hip-joint dependent on tubercular abscess of the neck of the femur, which had given way into the joint, I was able to scrape out the abscess cavity through the anterior incision, and obtain primary union. The operation took place more than eighteen months ago, and the joint continues perfect. ${ }^{5}$ In Case 4 it did not seem advisable to attempt primary union, for there was a fear that if much exudation took place it would, if unable to escape externally, force its way into the knee, and so establish a communicacion between the joint and the large cavity, a complication not unlikely to render the operation useless.

CASE 5. Alscrss of the os calcis -..It is not necessary to detail this cale $\mathrm{a}^{\mathrm{r}} \mathrm{any}$ great length. The patient, $\mathrm{A} . \mathrm{B}-$, aged seventeen, was brought to me at the Royal Free Hospital on March 30th, 1889, with a large swelling of the left foot. This bad been noticed for nine months, and had slowly in. creased, with absolutely no pain from the commencement. She knew of no cause. There was a large chronic abscess ocenpying the inner aspect of the posterior part of the os ca!cis, and extending from that for some distance along the inner border of the tendo Achillis. A curved incision into this gave exit to a quantity of cheesy pus, and on the surface of the bone was a circular opening about the size of a threepenny piece, with smooth rounded edges from which more caseous pus came. A gonge easily cut away the wall of a cavity, which was then found to be about the size of a chestnut, situated between the epiphysis and the body of the bone. A small caseous sequestrum, about the size of a hazel nut, was lying loose in the cavity. This was removed, the cavity scraped out with a sharp spoon, washed out with chloride of zinc and with perchloride solution, silk stitches were passed, the cavity plugged with strips of gavze which bad been dipped in iodoform and glycerine emulsion, lint and wool applied, and a plaster splint to keep the foot still. About thirty-six honrs afier the operation the plugs were removed, the stitches tied, and a tube inserted. The wonnd slowly filled up, and the patient left on April $26 \mathrm{r}$. There was still some cedema of the scar, but no pain. She returned in the following Jaly with some pain in the fost, but it subsided ander treatment, and until a month ago she did not present herself again, having been able to do her work as a servant; she then came with pain below the inner malleolus, rather worse at night; the signs were not, however, very definite, and quickly subsided under treatment. The unusual thing about this case was the complete absence of pain, although much of the os calcis was destroyed and the place of the bone taken by pus and caseating products. The only evidence of disease of the bone before operation was the widening of the bone, which was apparent in comparing the two sides. The process was a tubercular one, and the formation of the abscess took place in a part compusf d of cancellous bone tissue.

Harley-street, $W$.

\section{CAN INJURY PRODUCE LOBAR PNEUMONIA?}

BY JOHN A. MACDOUGALL, M.D., F.R.C.S.E., HONORARY CONSLTTING SCRGTON TO THE CUMBERLAND INFIRMARY.

THkRE is, perhaps, no disease which has a more broadly written etiology than pneumonia, and certainly none whose probable cansation has so widened and grown with advance in the science of medicine. Certain causes there are which, so far as clinical observation can guide us, stand as fairly well determined; but there are others to which doubt still attaches, and regarding which widely different opinions have been expressed. The number of surgeons is small, judging from contemporary literature, who have any doubt as to the direct relationship which injury bears to pneu. monia, and some physicians of high standing appear to hold a similar opinion; but there are others, like wise men of light and leading, whose strong belief is that ivjury to the chest cannot produce lobar pneumonia. In illustration of this present position I quote theopinion of two writers who nay justly be regarded as reliable exponents of surgical and medical

5 THE LANCET, vol. i. 1891, p. 1102 (Ibirl, vol. ii. 1890 , p. 820 . 